\title{
Clasificaciones, acuerdos y negociaciones: bases de la primera estadística internacional de enfermedades mentales (París, 1889)
}

\author{
Sandra Caponi (*) \\ $\left(^{*}\right) \quad$ Universidad Federal de Santa Catarina. Brasil. \\ sandracaponi@gmail.com
}

SUMARIO: 1.-Introducción. 2.-Propuestas para una clasificación unificada. 3.-Bases para una estadística internacional de alienados. 4.-Quételet y las estadísticas internacionales. 5.-Contar y cuantificar. 6.- Un nuevo modo de clasificar las enfermedades mentales se cristaliza.

RESUMEN: En este artículo se analiza la construcción de la primera clasificación internacional de enfermedades mentales, creada con el objetivo específico de permitir una estadística unificada de alienaciones. Esta clasificación fue discutida y aprobada en el Congreso Internacional de medicina mental realizado en París en agosto de 1889 , respondiendo a una solicitud que la Sociedad de Medicina Mental de Bélgica realizó cuatro años antes. Una lectura atenta de ese informe, nos ayudará a comprender el papel que entonces ocupaban las estadísticas y las clasificaciones en el campo de la medicina mental. Esto permitirá situar en perspectiva histórica un tema que aún hoy continúa siendo problemático, y analizar el momento en que, por primera vez, las clasificaciones psiquiátricas se articularon y se subordinaron a los estudios estadísticos comparativos.

PALABRAS CLAVE: Quételet, clasificar, cuantificar, estadística, psiquiatría.

KEY WORDS: Quételet; classification, quantification, statistics, psychiatry.

\section{Introducción $(*)$}

En el año 1885, se desarrolló en la ciudad belga de Amberes el I Congrès International de Phréniatrie al que concurrieron delegados de diferentes países de Europa. La Sociedad de Medicina Mental de Bélgica presentó

(*) Trabajo realizado en el transcurso de una estancia de investigación en la EHESS (Paris) con ayuda de una beca CAPES Senior. 
su propuesta de elaborar una clasificación internacional de enfermedades mentales capaz de servir de base para la realización de una buena estadística internacional de alienaciones. Esta sociedad debía recibir y centralizar la información, que cada país invitado estaba obligado a confeccionar y enviar en los cuatro años siguientes.

En el Congrés International de Médecine Mentale que debía celebrarse en París en agosto de 1889, la comisión internacional designada en Bélgica ya dispondría de elementos suficientes para presentar una síntesis de las posiciones encaminadas. Dicha síntesis fue sometida a discusión en el congreso de París para definir una clasificación única, obtenida por consenso, como referencia para todos los estudios estadísticos internacionales sobre enfermedades mentales.

Se analiza aquí ese debate con la finalidad de comprender la novedad que representa esa primera clasificación internacional de enfermedades psiquiátricas, enteramente concebida con fines estadísticos, desde el punto de vista de una historia epistemológica de la medicina mental.

En el transcurso de los cuatro años que sucedieron al congreso de Amberes, diversos países enviaron sus propuestas de clasificación, entre ellos: Alemania, Gran Bretaña, Bélgica, Estados Unidos, Rusia, Suecia, Italia, Dinamarca, y América del Sur, con una propuesta que unificaba a los delegados de Brasil, Chile, Perú, Argentina y México, allí presentes. Francia no envió propuesta de clasificación, aun cuando varios países habían tomado a la psiquiatría francesa como modelo para organizar sus propuestas y Francia fuera la sede del congreso internacional.

La Société Médico-psychologique de París había designado una comisión para que unificara los diversos abordajes existentes en la psiquiatría francesa. Pero esta comisión compuesta por Valentin Magnan, Paul Garnier, y M. Christian no logró, después de un largo debate de dos años ${ }^{1}$, llegar a un acuerdo con los miembros de la Sociedad que defendían criterios de clasificación diferentes a los que ellos proponían. Algunos creían que debía privilegiarse una clasificación etiológica, otros preferían una nosología basada en la anatomía patológica, otros privilegiaban la evolución de la enfermedad, y la mayoría coincidía en que toda nosografía debería integrar la sintomatología como un recurso necesario aunque no suficiente. Los

1. Garnier, Paul. Classification des maladies mentales. Annales Médico-Psycologiques. 1888; 8: 454-468. 
pormenores de este debate y los argumentos defendidos por cada una de las partes fueron objeto de estudio de un trabajo anterior ${ }^{2}$.

Jean-Christophe Coffin, en su libro La Transmission de la Folie, 1850$1914^{3}$, analiza el papel que la teoría de la degeneración ${ }^{4}$, fundamentalmente las patologías psiquiátricas propuestas por Benedict August Morel $^{5}$ y Valentin Magnan ${ }^{6}$ con el nombre de enfermedades heredo-degenerativas, ocupó en ese acalorado debate.

La sesión de apertura del Congreso internacional de medicina mental realizado en París en 1889, se denominó Des Bases d'une bonne statistique international des Aliénés. Rapport sur les travaux des délégués de la Commission internationale nommée au Congrès d'Anvers en $1885^{7}$. El análisis de dicho informe permite entender ese momento en que, por primera vez, las clasificaciones psiquiátricas se articulan y se subordinan a los estudios estadísticos.

Este congreso se inscribe en el punto de confluencia de dos cuestiones clave para comprender el proceso de institucionalización y universalización de la psiquiatría como saber y como profesión: el uso de las estadísticas en el campo de las enfermedades mentales y el proceso de internacionalización de la medicina mental que se dará a través de acuerdos tendientes a la normalización de un lenguaje científico en el campo de la psiquiatría. Se propone allí la estandarización de una nomenclatura unificada destinada

2. Caponi, Sandra. Para una estadística universal: un debate sobre la primera clasificación internacional de enfermedades mentales (1888-1889). Frenia. 2011; 11: 67-88.

3. Coffin, Jean-Christophe. La transmission de la folie, 1850-1914. Paris: Ed. Harmattan; 2003, p. 164-182.

4. A propósito de la teoría de la degeneración ver: Huertas, Rafael. Madness and degeneration: From «fallen angel» to mentally ill. History of Psychiatry.1992; 3: 391-341; Pick, Daniel. Faces of degeneration. A European disorders, c. 1848-c. 1918. Cambridge: Cambridge University Press; 1989. Serpa, Octavio. O degenerado. Historia, Ciência e saúde-Manguinhos. 2010; 17 (2): 447-474; Huertas, Rafael; Campos Marín, Ricardo. La teoría de la degeneración en España. In: Glick, Thomas; Ruiz, Rosaura; Puig-Samper, Miguel, eds. El darwinismo en España e Iberoamérica. México: Ed. UNAM; 1999, p. 231-348.

5. Morel, Benedict August. Traité des dégénérescences physiques, intellectuelles et morales de l'espèce humaine et des causes qui produisent ces varieties maladives. Paris: Bailliére; 1857.

6. Magnan, Valentin. Recherches sur les centres nerveux. Alcoolisme, folie des héréditaires dégénérés. Paris: Masson; 1893.

7. Morel, Jules. Des Bases d'une bonne statistique internationale des aliénés. Rapport sur les travaux des délégués de la comission internationale nommée au Congrès d’Anvers, en 1885. In: Ritti, A., ed. Congrès International de Médecine Mentale, tenu a Paris, du 5 au 10 août 1889. Paris: Masson; 1889, p. 44-52. 
a futuros estudios estadísticos y comparativos entre los diferentes países participantes.

Como destacan Olagüe de Ros et al., «el elemento clave sancionador de criterios normalizadores, tanto terminológicos como mensurativos, fueron las reuniones y los congresos internacionales en los diferentes campos científicos» ${ }^{8}$. Así, el congreso internacional de 1889 , puede ser visto como un verdadero campo de batalla donde se dirimieron disputas y rivalidades nacionales. Crear un acuerdo para definir una nomenclatura común sería considerado como el primer paso para consolidar una psiquiatría internacional unificada, condición primera para la realización de estadísticas comparadas. Las informaciones obtenidas se transformarían en auxilio para reforzar las identidades nacionales, fortalecer las ideologías colonialistas, e intentar responder a ese problema que reaparece de forma recurrente: entender si, en las sociedades industriales, la enfermedad mental tiende o no a aumentar 9 .

El recurso a las estadísticas había sido ampliamente utilizado en el campo de la medicina a partir de 1830 , como explican Murphy ${ }^{10}$, Rosen ${ }^{11}$ y Ackerknecht ${ }^{12}$ para referirse a la estrecha relación existente entre problemas médicos y conflictos sociales. Estudios como los de Villermé y de William Farr ${ }^{13}$, ponen en evidencia las complejas articulaciones existentes entre enfermedades y condiciones materiales de existencia. Sin embargo, al mismo tiempo en que las estadísticas permiten desnudar los efectos de la pobreza sobre la salud de las poblaciones, como afirma Martínez-Pérez,

«en la década de 1830 los métodos estadísticos que los médicos aplicaron a los asuntos de salud pública llegaron a convertirse en una herramienta de primer orden en los intentos oficiales por controlar todo lo que pudiera representar un motivo de desorden social $»^{14}$.

8. Olagüe de Ros, Guillermo et al. Internacionalismo y ciencia. Las bases sociocientíficas del movimiento documental europeo. Dynamis. 1997; 17: 317-340 (334).

9. Ackerknecht, Erwin. Historia de la psiquiatría. Buenos Aires: Eudeba; 1962.

10. Murphy, Terence. Medical knowledge and statistical methods in early nineteenth-century France. Medical History. 1981; 25: 307-308.

11. Rosen, Georges. A history of public health. New York: MD Publications; 1958.

12. Ackerknecht, n. 9.

13. Villermé, Louis René. De la santé des anciens ouvriers employés dans les fabriques de soie, de coton et de laine. Paris: BNF; 1829. Farr, William. Vital statistics: Memorial volume on selections from reports and writings. London: Bulletin of the World Health Organization; 2000: 78-81.

14. Martínez-Pérez, José. Suicidio, crisis política y medicina mental en la Francia del siglo XIX. Frenia. 2001; 1 (2); 39-65. 
La medicina mental se ocupará de algunos de esos factores considerados causa de desorden social, multiplicándose estudios referidos al aumento de alienados en los asilos, al alcoholismo y al suicidio. Sin embargo, los estudios estadísticos comparativos de enfermedades mentales se encontraban limitados, a fines del siglo XIX como en tiempos de Pinel, por la ambigüedad de las clasificaciones psiquiátricas existentes.

Los estudios de estadística psiquiátrica que habían sido iniciados por Pinel y continuados por Esquirol en el interior de los asilos psiquiátricos, contribuyeron a la profesionalización de la psiquiatría en las primeras décadas del siglo XIX. Pinel desarrolló diversos estudios estadísticos en la Salpêtrière ${ }^{15}$ analizando cuatro formas de manifestación de la alienación mental: manía, melancolía, demencia e idiotismo. Esquirol, a quien se le debe el proyecto de ley promulgado en Francia en 1838 sobre los asilos psiquiátricos, profundizó y amplió los estudios estadísticos sobre enfermedades mentales, siendo reconocido como un destacado estadístico ${ }^{16}$.

Esquirol parte de la clasificación propuesta por Pinel, diferenciando cinco grandes clases: manía, lipemanía, idiotismo, monomanía y demencia. Esta clasificación se convertirá en una referencia fundamental para la psiquiatría del siglo XIX, dando lugar a un largo debate en torno a la ambigüedad y al alcance del concepto de monomanía ${ }^{17}$.

Al mismo tiempo, existían serias restricciones sobre el modo de realización de las estadísticas psiquiátricas, limitadas a contar y registrar el número de sujetos ingresantes al asilo, el tiempo de permanencia, las curas registradas, los decesos y los egresos. En general las muestras de las que partían esas estadísticas eran pequeñas y poco representativas.

Aunque los estudios sobre la probabilidad se iniciaron con Laplace ${ }^{18}$ en 1812, las estadísticas médicas se mantuvieron, por mucho tiempo, ajenas a esa teoría que ingresará al campo de la medicina con los estudios

15. Pinel, Philippe. Traité Médico-Philosophique sur l'aliénation mentale. 2. ${ }^{\text {a }}$ ed. Paris: Brosson; 1809. Esquirol, Jean-Etienne-Dominique. Des maladies mentales considérées sous les rapports médical, hygiénique et médico-legal. Paris: Chez- Baillière; 1838; Woods, Evelyn; Carlson, Eric. The psychiatry of Philippe Pinel. Bulletin of the History of Medicine. 1961; 35: 14-25.

16. Ackerknecth, n. 9.

17. Déricq, Lucien. Leçon d'ouverture du cours de M. Magnan sur les maladies mentales. Annales médico-psicologiques. 1885; 2: 310-314 (311).

18. Laplace, Pierre-Simon. Essai philosophique sur les probabilités. Oeuvres complètes. Paris: Gauthier-Villars; 1878-1912. 
de Quételet en $1835^{19}$. Sin embargo, en el campo de la salud mental, las estadísticas psiquiátricas continuaron, por mucho tiempo, siendo compilados por observación y numeración. Es en ese contexto que debe situarse el análisis del Congreso Internacional de Medicina Mental de 1889 que será aquí desarrollado.

\section{Propuestas para una clasificación unificada}

El Informe denominado Des Bases d'une bonne statistique international des Aliénés fue presentado por Jules Morel, representante de la Sociedad Belga de medicina mental. Resume las clasificaciones enviadas por los países participantes del Congreso de Amberes ${ }^{20}$, entre los años 1885 y 1889, y presenta una propuesta de clasificación que contempla las anteriores.

Morel inició la presentación de su Informe, recordando la tarea que se les había asignado. La comisión debía confeccionar una clasificación de enfermedades mentales, destinada a una estadística internacional, lo más simple posible y limitada a un número reducido de tipos mórbidos. Esa clasificación surgiría, por consenso, de la comparación entre las clasificaciones enviadas.

El primer país en responder a ese pedido fue Alemania que presentó seis grupos mórbidos: tres denominados «alienación mental» (simple, con parálisis y con epilepsia), seguidos por «imbecilidad»y «delirium», y finalmente la clase, difícil de comprender, de «no alienados». Esta clasificación sería rápidamente rechazada por considerar que no incluía, ni permitía integrar, las enfermedades conocidas por los alienistas del mundo. A continuación se analizaron las propuestas de Suiza y de Austria-Hungría, que también diferenciaban cinco grupos, considerados más inteligibles pero insuficientes. La comisión entendió que estas clasificaciones operaban «a nuestros ojos, una simplificación muy grande, agrupando formas mórbidas que merecerían estar separadas desde el punto de vista de la estadística» ${ }^{21}$.

19. Quételet, Adolphe, Sur I'homme et le développement de ses facultés. Paris: Fayard; 1991 [original: Paris: Bachelier Imprimeur; 1835].

20. La comisión que elabora el informe presentado por J. Morel, estaba compuesta por: Hack-Tuke representando a Gran Bretaña, Ramaer a Holanda, Steenberg a los países escandinavos, Magnana Francia, Willea Suiza, Verga a Italia, Guttstadta Alemania, Mierzejewskia Rusia, Clark-Bell a América del Norte y Solá a la América meridional. Morel, n. 7, p. 44.

21. Morel, n. 7, p. 46. 
Ambas repetían el modelo de Alemania, cuya propuesta se limitaba a insistir en el mismo esquema utilizado para realizar sus estadísticas en los hospitales de alienados.

Dinamarca, Suecia y Noruega presentaron un cuadro con siete grupos con sus formas mórbidas correspondientes. Entre los grandes grupos aparecía la denominación «psicosis degenerativas» que incluía monomanías, locura periódica, hipocondría y locura moral. Para la comisión esta clasificación tenía un mérito mayor que las otras, lo que indicaría que los delegados habían comprendido la necesidad de crear una tipificación capaz de ser utilizada por todos los alienistas del mundo. Rusia, por su parte, se limitó a presentar una larga lista de patologías mentales sin ningún tipo de organización o jerarquía, que fue rápidamente descalificada por la comisión.

Un año más tarde, otros países enviaron sus propuestas. Italia y los países escandinavos, elaboraron un cuadro donde se presentaban nueve grupos, precedidos por la denominación «psicosis» (congénita, simple, aguda, consecutiva, primitiva, convulsiva, tóxica, otras formas psicopáticas), diferenciando las formas mórbidas que corresponden a cada grupo. Se consideró que esta clasificación poseía un número excesivo de grupos y entidades mórbidas, tipos patológicos mal definidos, como las «psicosis crónicas o convulsivas», en el caso de Italia, y las «psicosis degenerativas», en el caso de Escandinavia, pudiendo dar lugar a interpretaciones divergentes.

Las clasificaciones propuestas por Gran Bretaña y Estados Unidos, semejantes entre ellas, fueron consideradas como propuestas válidas sin ninguna objeción ${ }^{22}$.

Así, exceptuando la propuesta de Gran Bretaña, todas las otras fueron rechazadas, unas por ser amplias, otras por ser sintéticas, unas porque presentan sub-grupos, otras porque se limitaban a presentar grandes grupos. Lo mismo ocurrió con la propuesta de Holanda de tres grupos patológicos: «Delirios», «procesos psicopáticos» $\mathrm{y}$ «estados psicopáticos» ${ }^{23}$. Su única novedad fue la «locura sexual» con dos sub-grupos: ninfomanía y satirismo. América Meridional contó con siete delegados: dos de México y dos de Brasil, y un delegado por Perú, Chile y Argentina. Presentaron una clasificación que distinguía seis grupos: «Manía y delirio», seguido de «lipemanía, locuras tóxicas, orgánicas, neuropáticas y mixtas». Esta 
propuesta fue descalificada, argumentando que «esta clasificación difiere mucho de las precedentes y no es necesario que insistamos en ella» ${ }^{24}$. Más tarde Cabred, delegado de Argentina, presentó otra clasificación que también fue rápidamente desautorizada. Como afirma Coffin ${ }^{25}$, la clasificación presentada por los sudamericanos fue considerada obsoleta, quizás por el uso de la denominación lipemanía.

\section{Bases para una estadística internacional de alienados}

Después de analizar las alternativas presentadas por los delegados internacionales, llegaría el momento de la síntesis final elaborada por la Société de Médecine Mentale de Belgique. Ésta propuso una clasificación semejante a la que había sido sometida a discusión en el congreso de 1885. La clasificación inicial contaba con ocho formas mórbidas y pocas subdivisiones ${ }^{26}$. La semejanza con la clasificación a la que se llegaría después de cuatro años de discusión con los más diversos países del mundo, no deja de ser sorprendente.

La Société de Médecine Mentale de Belgique presentó en 1889 una propuesta de síntesis basada en la convicción de que la división en grandes grupos debía ser abandonada, siendo más apropiada para una estadística internacional una clasificación de formas mórbidas simples. Concluía que toda clasificación etiológica debía ser excluida, en la medida en que podía llevar a confusiones en el momento de elaborar las estadísticas.

De ese modo, los alienistas del mundo entero aceptaron, como la comisión belga había propuesto en 1885, que una buena clasificación de enfermedades mentales debía basarse exclusivamente en formas clínicas. Aparentemente, al optar por una clasificación simple basada en síntomas, se cerraban los debates interminables dedicados a definir un criterio privilegiado de clasificación de alienaciones. Se trataba de una tregua momentánea, ya que la disputa en torno a los criterios de clasificación continuó tan viva como antes. Pero, el objetivo de crear una lista simple de patologías que

\footnotetext{
24. Morel, n. 7, p. 50.

25. Coffin, n. 3, p. 176.

26. Presentaba los siguientes grupos: «ldiotismo, demencia simple, manía y melancolía, delirio agudo, locura moral, locura circular, alienaciones mentales (con parálisis, epilepsia, histeria o tumores) y alienación por intoxicación». Garnier, n. 1, p. 463.
} 
sirviera como base para una estadística internacional de enfermedades mentales, ya se había logrado.

La Société de Médecine Mentale de Belgique propuso, en la síntesis final, once formas mórbidas: «Manía, melancolía y demencia aguda, locura periódica, locura sistemática progresiva, demencia posterior a una enfermedad mental, demencia posterior a una enfermedad local del cerebro, parálisis general, locuras neuróticas (histeria, epilepsia e hipocondría), locuras tóxicas, locura moral e impulsiva, idiotismo e imbecilidad ${ }^{27}$. Una clasificación semejante a la presentada en Amberes con pocas diferencias: la introducción de la categoría "parálisis general» y la substitución de las «alienaciones mentales vinculadas a histeria, epilepsia y tumores cerebrales» por la clase «locuras neuróticas». Se llegaba así a una clasificación simple, basada exclusivamente en criterios sintomáticos.

La Sociedad Belga parecía haber olvidado que, desde 1857, fecha en la que Morel publicó su Tratado de degeneración ${ }^{28}$, el problema de la clasificación etiológica formaba parte de la agenda de debate de los alienistas del mundo. Esa posición fue recuperada y defendida en el congreso por varios delegados, entre ellos Cabred ${ }^{29}$, representante de Argentina. Siguiendo la teoría de la degeneración de Morel, proponía cuatro grandes grupos patológicos que se diferencian por sus causas: «locura vesánica, neuropática, tóxicas, orgánicas y degenerativas». Concluida la extensa exposición de Cabred, Charpentier cerró cualquier posibilidad de debate: «Debo destacar - afirmaba- que no es posible considerar esta clasificación que se acaba de proponer como una clasificación científica de enfermedades mentales» ${ }^{30}$.

$\mathrm{Ni}$ el criterio etiológico, ni el criterio anatomopatológico, serían considerados válidos en este debate. Las delegaciones de Holanda, Suiza e Italia habían destacado la importancia de las Psicosis orgánica y de los criterios anatomopatológicos. Pero, aunque las formas mórbidas «parálisis general» $\mathrm{y}$ «demencia posterior a una enfermedad local del cerebro», encontraron un lugar en la clasificación de Bélgica, la localización anatomopatológica no sería considerada un criterio válido de clasificación.

\footnotetext{
27. Morel, n. 7, p. 51.

28. Morel, n. 5.

29. Cabred, Domingo. Discussion sur le Rapport des travaux des délégués de la Comission internationale nommée au Congrès d'Anvers, en 1885. In: Ritti, n. 7, p. 53-56.

30. Charpentier, Alphonse, Discussion sur le Rapport des travaux des délégués de la Comission internationale nommée au Congrès d'Anvers, en 1885. In: Ritti, n. 7, p. 11-58 (57).
} 
Otros dos criterios fueron ignorados: la clasificación por evolución mórbida defendida por Magnan ${ }^{31}$ y los teóricos de la degeneración ${ }^{32}$, y la clasificación mixta que había sido apasionadamente reivindicada por la comisión que representaba a la Société Médico-psychologique de Francia ${ }^{33}$. El criterio mixto propuesto por esa Comisión, precedida por Garnier y Magnan, fue rechazado por la mayoría de los alienistas franceses ${ }^{34}$. Dos años de acalorados debates en la Société Médico-psychologique siguieron a esa propuesta sin que se pudiera alcanzar un acuerdo ${ }^{35}$.

La Société de Médecine Mentale de Belgique privilegió un único criterio centrado en las bases clínicas. De manera que la clasificación sintomática defendida por Esquirol, criticada por los alienistas franceses de la segunda mitad del siglo XIX ${ }^{36}$, como poco confiable, elástica y ambigua, se transformó en el criterio hegemónico para una clasificación de enfermedades mentales destinada a la construcción de una buena estadística internacional.

Como afirmaba Déricq ${ }^{37}$, al presentar el curso de Magnan de 1885, uno de los métodos existentes para estudiar las patologías mentales aludía al gran complejo sintomático por el que se manifiestan las enfermedades. En este caso se separa el síntoma que más se destaca en un cuadro patológico, y se lo eleva a la categoría de entidad mórbida, como en la agorafobia o la cleptomanía. Se consideraba que ese método, exclusivamente fundado en formas clínicas, era insuficiente para construir una nosología que pudiera considerarse científica o al menos satisfactoria.

Es verdad que, independientemente de la intempestiva intervención de Charpentier ${ }^{38}$ contra el representante de Argentina, la Comisión de Amberes nunca se había propuesto el objetivo de crear una clasificación científica de enfermedades mentales. No ignoraba que la clasificación propuesta "presentaba lagunas y que, desde un punto de vista científico,

31. Magnan, n. 6.

32. Garnier, n. 1.

33. Garnier, Paul. Classification des Maladies Mentales (suite). Annales Médico-Psycologiques. 1889; 9: 457-470 (474).

34. Luys, Jules. Classification des maladies mentales. Annales Médico-Psychologiques. 1889; 10: 264-274. Ball, Benjamin. Classification des maladies mentales. Annales Médico-Psychologiques. 1889; 10: 112-119.

35. Coffin, n. 3; Falret, Jules, Chronique du Congrès International de Médecine Mental. Annales Médico-Psycologiques. 1889; 9: 177-186 (179).

36. Morel, n. 5; Magnan, n. 6; Falret, n. 35.

37. Déricq, n. 17.

38. Charpentier, n. 30. 
resultaba imposible exhibir una obra completa ${ }^{39}$. Su objetivo era mucho más modesto. Se trataba de dar una solución, lo más simple posible, a la urgente necesidad de crear una estadística internacional que pudiera suplir la fragilidad de los estudios estadísticos realizados.

Contrariamente a lo que podía imaginarse, llegar a una clasificación internacional científica de enfermedades mentales no era el objetivo perseguido por la comisión belga en el congreso de 1885. La clasificación unificada no era más que un medio, un instrumento que permitiría alcanzar una finalidad mayor: conferir a la estadística de enfermedades mentales un nuevo estatuto epistemológico. Lo que se buscaba no era crear una clasificación más rigurosa, sino una buena estadística, esto es una estadística confiable. Y la clasificación era el paso inicial y necesario para comenzar a hablar un idioma común. Como afirmaba J. Morel:

«al aceptar estas bases internacionales, aunque la obra de la estadística no podrá alcanzar un valor científico absoluto, al menos será fecunda en enseñanzas y dará un gran servicio a las naciones civilizadas ${ }^{40}$.

La larga espera de cuatro años para definir, por consenso, una clasificación simple y no científica de enfermedades psiquiátricas parecía estar más que justificada. Ningún criterio, entre los utilizados y defendidos hasta entonces, constituía una base científica sólida como para validar una clasificación única. Sucesivamente fracasaron los intentos por encontrar el fundamento de las clasificaciones psiquiátricas en bases anatomopatológicas, etiológicas, evolutivas o mixtas. Así, el criterio más simple y frágil de clasificación, y el que más ataques recibiera, el criterio sintomático sería el privilegiado, sin reservas, por la comisión belga.

Lo que esa comisión pretendía no era crear una clasificación consistente, sino construir un nuevo modo de entender las enfermedades mentales donde las estadísticas ocupaban un lugar central. Existía un consenso en relación a la utilidad del criterio sintomático como auxilio necesario, aunque no suficiente, para definir las clasificaciones de enfermedades mentales. Los defensores de los otros criterios afirmaban que las formas clínicas no podían ser desatendidas, pero reconocían que limitarse a esa información podía llevar a errores. Por otra parte, los estudios estadísticos referidos a

39. Morel, n. 7.

40. Morel, n. 7, p. 53. 
enfermedades mentales existentes hasta ese momento no habían dejado ninguna contribución relevante ${ }^{41}$.

Tomando como base una clasificación internacional unificada, simple y basada en síntomas, sería posible comparar datos provenientes de diferentes países referidos al número de alienados, de curas conseguidas, de aumento o de disminución de formas mórbidas. Un cuidadoso registro y cuantificación de estos datos, permitiría que la estadística de enfermedades mentales se transformara en una instancia de validación para el campo de la psiquiatría.

De ese modo, la estadística dejaba de ser un auxilio secundario para los alienistas. Tanto la Société de Médecine Mentale de Belgique como los delegados presentes compartían la misma confianza en el poder explicativo de las estadísticas. Parecía factible que en el futuro, y frente al fracaso de los intentos por localizar las patologías mentales en el cerebro, las estadísticas pudieran llegar a convertirse en el principal instrumento de validación de la medicina mental.

\section{Quételet y las estadísticas internacionales}

Lo que Charpentier no podía imaginar era que, en esas circunstancias, su posición era tan obsoleta y anticuada como la propuesta de Cabred que tan duramente había criticado ${ }^{42}$. Si Cabred ${ }^{43}$, de manera extemporánea, presentaba como novedosa una clasificación idéntica a la que B. A. Morel había defendido cincuenta años antes, Charpentier cometía un error semejante al reclamar validez científica a la clasificación internacional que allí se estaba discutiendo. J. Morel ya lo había enunciado claramente y Semal, otro delegado belga, se encargaría de repetirlo:

«la misión de la Société de Médecine Mentale de Belgique está claramente definida. Ella no tiene la pretensión de presentar una clasificación científica de enfermedades mentales, sino la de proponer a votación una lista de tipos

\footnotetext{
41. Morel, n. 7, p. 52.

42. Charpentier, n. 30.

43. Cabred, n. 29.
} 
mórbidos lo más claros e indiscutibles posibles, que pueda servir de base para una estadística internacional de la locura» ${ }^{44}$.

A esta afirmación le sucedería la de Clark-Bell, representante de Estados Unidos, quien después de una acalorada defensa de la posición presentada por Bélgica, destacó las analogías con la clasificación utilizada en su país y su relevancia: «considero este voto como el más importante que se emitirá en este Congreso Internacional» ${ }^{45}$.

Algunos miembros de la Sociedad Médico-psicológica de París pidieron la substitución de la categoría «locura moral» por «locura hereditaria o de los degenerados» ${ }^{46}$, pero la petición no fue aprobada. Finalmente, y para concluir la sesión, se procedió a la votación de la clasificación presentada por la Sociedad Belga de Medicina Mental, que fue aprobada por mayoría. Eso significaba un compromiso de que todos los países presentes adoptarían esa clasificación para elaborar sus estadísticas, consolidando el proceso de internacionalización de la psiquiatría.

Pero, ¿por qué razón atribuir tanta relevancia a una simple lista de tipos mórbidos, más o menos discutible, a la que se había llegado por una votación? Para responder a esta pregunta, para entender la importancia de este debate deberemos buscar una respuesta ya no en el campo de la psiquiatría sino el campo de la historia de las estadísticas aplicadas a los hechos sociales.

El hecho, aparentemente trivial, de que la propuesta de una estadística internacional de enfermedades mentales fuese presentada por Bélgica, no era un dato menor. Bruselas había sido la sede del I Congrès International de Statistique, realizado en 1853 por iniciativa de Adolphe Quételet ${ }^{47}$, dando lugar a la posterior fundación del Instituto Internacional de Estadística de Londres en 1885. El objetivo de ese Congreso era doble, centralizar las estadísticas en los diferentes países y posibilitar la comparación de los datos obtenidos. Esto exigía uniformizar las técnicas de compilación de

\footnotetext{
44. Semal, M. Discussion sur le Rapport des travaux des délégués de la Comission internationale nommée au Congrès d'Anvers, en 1885. In: Ritti, n. 7, p. 57.

45. Clark-Bell, M. Discussion sur le Rapport des travaux des délégués de la Comission internationale nommée au Congrès d'Anvers, en 1885. In: Ritti, n. 7, p. 57.

46. Ball, Benjamin, Discussion sur le Rapport des travaux des délégués de la Comission internationale nommée au Congrès d'Anvers, en 1885. In: Ritti, n. 7, p. 58.

47. Quételet, n. 19.
} 
datos con la finalidad de facilitar la comparación ${ }^{48}$. De acuerdo a Cussó, en el momento de presidir el I Congrès International de Statistique, Quételet definió los siguientes objetivos:

«buscar introducir unidad en las estadísticas oficiales que publican los gobiernos, y transformar a esos datos en comparables. Los trabajos seguramente serán más fáciles cuando sean definidas las bases generales a las cuales deberemos ajustarnos, cuando sean adoptadas en los diferentes países nomenclaturas y clasificaciones uniformes, esta especie de lenguaje universal, al simplificar los trabajos les asegurará mayor importancia y solidez. (...) El medio más seguro para llegar a la unidad deseada parece ser la creación, en cada Estado, de una Comisión general de estadística» ${ }^{49}$.

Ese congreso estableció recomendaciones para la realización de censos en diferentes países, insistió en la necesidad de un registro centralizado y minucioso de edad, sexo, nacimientos y muertes. Se sugirió introducir informaciones como territorio, agricultura, industria, comercio, clases laboriosas, educación, criminalidad y locura. Esa no era una tarea simple, por esa razón cuando se reunió el II Congrès International de Statistique en San Petesburgo, en 1872, muchas de estas informaciones aún no estaban disponibles. La primera dificultad para conseguir ese objetivo era la descentralización de los datos, como ocurría en Francia ${ }^{50}$. La Société de Statistique de la France creada en 1860, enfrentaba en 1880, el problema de la comparabilidad y centralización de los datos enviados por los diferentes departamentos, un problema a ser resuelto antes de poder comparar registros internacionales ${ }^{51}$.

Adolph Quételet ${ }^{52}$, fue una figura clave para comprender los motivos que condujeron a la Société de Médecine Mentale de Belgique a discutir las

48. Desrosières, Alain. Adolphe Quételet. Courrier des statistiques. 2002;10: 2-8.

49. Cussó, Robert. La quantification internationale à la lumière de la SSP et des Congrès Internationaux de statistique. Electronic Journal for History of Probability and Statistiques. 2010; 6 (2):

2-19, p. 10-11. Cussó hace referencia a parte de las Compte-Rendu du I Congrès International de Statistique de 1853, p. 140.

50. Cussó, n. 49.

51. Rabinow, Paul. Une France si moderne. Naissance du social 1800-1950. Paris: Buchet-Chastel; 2006. Jorland, Gerard. Une société asoigner. Hygiène et salubrité publiques en France au XIX siècle. Paris: Ed. Gallimard; 2010.

52. Una amplia bibliografía ha sido dedicada a resaltar la relevancia del pensamiento de Adolphe Quételet, que además de ser astrónomo, fue también estadístico, demógrafo y sociólogo, destacamos aquí los textos clásicos: Halbwachs, Maurice. La Théorie de l’homme moyen: 
bases para una estadística internacional de alienaciones. Quételet estaba interesado en la comparación de los más diversos datos, no entre localidades, sino entre países, respetando la llamada ley de los grandes números ${ }^{53}$. Su propuesta era estudiar los fenómenos sociales y biológicos más diversos, desde la altura y el peso hasta el crimen y el suicidio, en términos tan objetivos e indiscutibles como los utilizados por los astrónomos para establecer la posición de una estrella. Estaba convencido de que era posible medir los hechos de los que se ocupaba la física moral, con la precisión con que se medían las orbitas estelares. Fue Quételet (1796-1874), cuya influencia se hizo sentir en diversas partes del mundo, quien abrió las puertas para comenzar a pensar los hechos y sufrimientos humanos en términos matemáticos.

Para Quételet, no solo era posible definir el número medio de crímenes, altura, nacimientos o casos de locura, sino también el grado de dispersión o los límites de variabilidad que podían ser considerados aceptables, tanto para más como para menos, en cada caso. Los hechos sociales tenían una asombrosa regularidad, presentando variaciones más o menos significativas. Los que se mantuvieran dentro de límites de variabilidad aceptable serían considerados normales, los que escapaban de esos límites representaban un desvío patológico o una tendencia hacia un estado anormal ${ }^{54}$.

Para Quételet «Los márgenes de variación (para más y para menos) son más restringidos en un pueblo, cuanto más éste se aproxima a la perfección» ${ }^{55}$. Como consecuencia, las sociedades civilizadas tenderían a aproximar los límites entre los cuales variaban las diferentes características humanas, existiendo menor diversidad entre sus miembros y prevaleciendo características físicas y morales normales, propias del hombre medio. El desarrollo de la medicina estimulaba el uso de nuevas tecnologías como las cirugías, las vacunas y las medidas de higiene permitían el desarrollo normal de todas las funciones humanas ${ }^{56}$. Otras medidas, como el control

essai sur Quételet et la statistique morale. Paris: Alcan; 1912, Frank H. Hankins. Adolphe Quetelet as statistician. London: King \& Son; 1908. Algunas referencias más recientes son: Desrosiéres, Alain. La politique des grands nombres: histoire de la raison statistique. Paris: La Découverte; 2010; Ball, Philip. Critical mass: How one thingleads to another. London: Arrow Books; 2005; Stigler, Stephen. Statistics on the table: The history of statistical concepts and methods. Cambridge: Harvard University Press; 1999.

53. Hacking, lan, La domesticación del azar. Barcelona: Ed. Gedisa; 1990, p. 143-171.

54. Halbwachs, n. 52; Quételet, n. 19.

55. Quételet, Adolphe, Du systeme social et des lois qui le régissent. Paris: Guillaumin; 1848, p. 38.

56. Quételet, n. 55, p. 260. 
de trabajo infantil o la buena alimentación contribuían a aproximar los límites entre los cuales oscilaba la normalidad en la sociedad, mientras que el grado de dispersión en relación a la media podría llegar a extremos en condiciones de gran desigualdad social.

En relación a la locura, la posición de Quételet era ambigua, suponía que las sociedades contaban con un número constante de casos, que se repetía con precisión año a año, ya fuese de crímenes, de suicidios, de nacimiento o de alienados:

«Podemos enumerar anticipadamente cuántos individuos mancharán sus manos con sangre de sus semejantes, cuántos serán falsificadores, cuántos envenenadores, con tanta precisión como podemos enumerar la cantidad de nacimientos y muertes que ocurrirán en una sociedad. La sociedad contiene en ella los gérmenes de todos los crímenes que se cometerán (...) Es ella quien prepara sus crímenes, el culpable no es más que el instrumento que los ejecuta ${ }^{57}$.

Los crímenes, como los nacimientos, o los casos de locura eran efecto de la organización social, de modo que solo era posible alterar la frecuencia con que se manifestaban estos hechos por cambios sociales globales y no por cambios en los comportamientos individuales ${ }^{58}$.

Pero la locura, a diferencia de otras enfermedades, era considerada por Quételet como efecto indeseable de las conquistas de la civilización. Asociaba la locura al desarrollo de las facultades intelectuales, de forma que cuanto más aumentaban estas facultades más aumentaba el número y la intensidad de esas patologías ${ }^{59}$. Varias referencias estadísticas reforzaban su tesis. Comparó diversos países, la cantidad de alienados, idiotas y locos registrados en cada uno, la distribución de alienados en la ciudad y en el campo. Comparó los meses en que ocurría la mayor cantidad de casos de alienación mental y de recuperación, la influencia de la edad, de las estaciones, del tipo de trabajo ${ }^{60}$.

Quételet presentó una descripción sumaria de las posibilidades de aplicación de la estadística a la alienación mental. Años más tarde, en el

\footnotetext{
57. Quételet, n. 55, p. 315. Hacking, n. 53.

58. Rabinow, n. 51; Sánchez Carrión Juan. Sociología, orden social y modelización estadística. Empíria. Revista de Metodología de Ciencias Sociales. 2000; 3: 49-71.

59. Quételet, n. 55, p. 389.

60. Quételet, n. 55, p. 392.
} 
Congrés International de Médecine Mentale de 1889, fueron retomados los mismos objetivos que él formuló en 1853: la necesidad de unificar las estadísticas oficiales que publicaban los diferentes gobiernos; la exigencia de que esos datos fuesen comparables; su interés por definir las bases generales para una buena estadística; la necesidad de adoptar nomenclaturas y clasificaciones uniformes en los diferentes países y la confianza en que la simplificación de los datos ayudaría a construir investigaciones sólidas.

Estos objetivos estaban presentes en la propuesta realizada por la Comisión Belga de medicina mental en el Congreso de 1885 y orientaron los trabajos de los delegados del Congreso Internacional de París de 1889. Sólo en ese marco podemos comprender ese largo debate de cuatro años, realizado por las sociedades de medicina mental de países tan diferentes como Rusia, Argentina, Estados Unidos o Perú, para poder crear una lista, sin pretensión de cientificidad, lo más simple posible y definida, después de una larga discusión, por votación.

\section{Contar y cuantificar}

Aun cuando fracasase el objetivo de la Société de Médecine Mentale de Belgique de crear una clasificación utilizada por todos los países civilizados ${ }^{61}$, en el congreso de París surgió un nuevo modo de pensar las estadísticas psiquiátricas que tendía a la internacionalización científica. Esta transformación parecía indispensable, pues, como se afirma en el informe, «sería inútil insistir en el hecho de que, hasta este momento, el estudio de las estadísticas de los alienados no ha sido en absoluto fructífera» ${ }^{62}$.

Desde los estudios de Pinel y Esquirol, las estadísticas psiquiátricas habían sido utilizadas de un modo similar. Se trataba de contar la cantidad de enfermos internados en los asilos de alienados, de saber cuántos eran hombres y cuántas mujeres, cuántas recuperaciones se habían conseguido y cuántos decesos ${ }^{63}$. Los estudios estadísticos se habían limitado a usar

61. Un ejemplo entre otros es la clasificación enunciada por Magnan y Legrain años más tarde. Magnan, Valentin; Legrain, Paul Maurice. Les dégénérés: état mental et syndromes épisodiques. Paris: Rueff; 1895.

62. Morel, n. 7.

63. Pinel, n. 15; Esquirol, n. 15; Falret, Jean Pierre. Recherches statistiques sur les aliénés, les suicides et les morts subites. Memoire Académie de Sciences. Paris: Masson; 1828; Jorland, n. 51. 
los registros de ingresos y egresos, para construir tablas de doble entrada indicando si, en un determinado asilo mental, había más hombres o mujeres, más viejos o jóvenes, o más muertes.

El Congrés International de Médecine Mentale de 1889 permite entender el momento en que ingresa al campo de la psiquiatría un nuevo modo de pensar las estadísticas, que ya no se limita a contabilizar casos de locura en asilos o prisiones. La operación de contar los casos de locura dejará lugar a la creación de un proceso de cuantificación de las alienaciones. Esta transformación, en apariencia poco significativa, exigirá la definición de nuevos procedimientos analíticos y la creación de un idioma común para realizar esas mediciones.

Esta nueva modalidad de estudios estadísticos supone dos operaciones igualmente importantes y sucesivas: clasificar y medir. Esto significa, establecer convenciones y transformar esas convenciones en números. Primero será necesario unificar las nomenclaturas, establecer clases y categorías aceptadas por todos los participantes del proceso de cuantificación, para luego, y solo entonces, poder comenzar el proceso de medición ${ }^{64}$.

Para Desrosières cuantificar significa «expresar y hacer existir bajo una forma numérica lo que antes era enunciado con palabras y no con números» ${ }^{65}$. Esta operación es diferente de la tarea de contar. Esta última, presupone la existencia de objetos reales a ser contados, sean manzanas o estrellas, o medidos, como la altura de los soldados o el ancho del río Paraná. La confusión que frecuentemente se hace entre cuantificar y con$\operatorname{tar}^{66}$ llevó a ocultar los arduos procesos de negociación que, en el campo de las ciencias sociales en general y en el de la psiquiatría en particular, precedieron al proceso de medición. Se tiende a olvidar que para poder medir y comparar es necesario crear previamente categorías o clases de comparación, denominadas por Desrosières, «convenciones previas de equivalencia» ${ }^{67}$. Crear estas convenciones implica establecer acuerdos y negociaciones que deben ser realizadas en espacios sociales determinados

\footnotetext{
64. Hacking, lan. Historical ontology. New York: Harvard University Press; 2004.

65. Desrosières, Alain. Pour une sociologie historique de la quantification. L'argument statistique. Paris: Mines; 2008, p. 10; Hacking, n. 64. Hacking, lan. Façonner les gens: le seuil de pauvreté In:. Beaud, J.-P.; Prévost, J.-G., eds. L'ère du chiffre: Systèmes statistiques et traditions nationales. Québec: Presses de l'Université de Quebec; 2000, p. 18-36.

66. Hacking, lan. Rewriting the soul: Multiple personality and the sciences of memory. Princenton: Princenton University Press; 1995.

67. Desrosières, n. 65, p. 12.
} 
tales como asambleas, congresos o reuniones científicas para ser considerados legítimos.

Las estrategias estadísticas de contar y cuantificar requieren procedimientos y métodos completamente diferentes. En el primer caso, se trata, como dijimos, de contabilizar registros que se refieran a objetos anteriores al procedimiento de medición. En el segundo caso, la enumeración será posterior a la creación de criterios previos de equivalencia que definen los contornos de los objetos a ser medidos. Estos dos procedimientos tienen un origen común en los estudios de Quételet. Por un lado, él trasladó el modelo de los grandes números y la idea de media estadística a los asuntos humanos. Leyes constantes, tan válidas como la ley de gravedad, permitían explicar la regularidad con que se manifestaban los hechos sociales. No solo la altura de los soldados, sino también el crimen y la locura eran hechos objetivos, anteriores a toda medición, que podían ser contados con los mismos procedimientos utilizados por las ciencias naturales. Para Desrosières:

«Las ciencias de la naturaleza han impuesto una epistemología de la medida. La ley de los errores ha inspirado a Quételet para construir su hombre medio. Esa metrología realista se impone como un modelo primero, del cual los estadísticos nunca han podido separarse completamente» ${ }^{68}$.

Pero también la cuantificación de hechos sociales surge de los trabajos de Quételet dedicados a definir indicadores macrosociales, como el índice de masa corporal o la propensión al crimen, como categorías a partir de las cuales podían ser realizadas mediciones y comparaciones. Para Quételet, estos indicadores no eran el resultado de convenciones sociales sino que surgían claramente por la observación de la constancia y regularidad con que aparecen los hechos en el mundo. Indicadores como la relación peso-altura reflejaban atributos reales que no podían ser directamente observados y que precisaban de esa mediación para poder ser contados. Esas «variables latentes» ${ }^{69}$ indican un fenómeno real pero inalcanzable por los procedimientos clásicos de medición.

En el proceso de cuantificación que se debatió en el congreso de 1889, los objetos a ser clasificados y medidos, suponían la existencia de patologías reales a las que no era posible acceder directamente. Pero esos objetos

68. Desrosières, n. 65, p. 160.

69. Desrosières, n. 65, p. 160; Hacking, n. 64. 
no se presentaban con la constancia y la regularidad que Quételet había observado en los hechos por él estudiados. Clasificaciones psiquiátricas y definición de síntomas parecían fluctuar sobre una arena movediza de incertezas. Así, y frente a la dificultad para poder crear indicadores a partir de regularidades observables, la Société de Médecine Mentale de Belgique presentaría otra estrategia de legitimación: definir por consenso una clasificación internacional unificada que sirviese como una convención de equivalencia, previa a la realización de las mediciones.

Ya no se trata de suponer, como en el modelo anterior, que los indicadores se refieren a una realidad oculta que ellos permitirían develar. Ahora, los indicadores ya no son pensados como resultado de regularidades sino de convenciones sociales, y su legitimidad estará directamente vinculada a la solidez de la inversión social realizada para producir esas clasificaciones ${ }^{70}$. Desrosières afirma que este modelo no se deriva, ni de las ciencias naturales, ni de las ciencias de la vida, sino del derecho y de los procedimientos utilizados en los parlamentos para establecer decisiones ${ }^{71}$.

\section{Un nuevo modo de clasificar las enfermedades mentales se cristaliza}

La Société de Médecine Mentale de Belgique supuso que, para crear una buena estadistica de alienaciones mentales, era necesario postular una instancia que antecede al proceso de medición, esto es, la creación de una clasificación unificada de alienaciones ${ }^{72}$. El procedimiento utilizado para crear esa clasificación articuló dos formas de definir indicadores y clases de equivalencia. Por un lado, se aproximó al modelo utilizado por las ciencias de la vida. Cuando J. Morel o Clark-Bell defendieron la clasificación propuesta por Bélgica lo hicieron utilizando argumentos provenientes del campo de la medicina: son los síntomas, las formas clínicas las que permiten identificar once patologías diferentes y reales que se ocultan a la simple observación, se trata de agrupamientos sintomáticos incuestionablemente tan reales como el cólera o la gripe.

Las clasificaciones no encontraron su legitimidad en lesiones anatomopatológicas específicas, como en la medicina interna, sino en un acuerdo

70. Christian, Mouhanna. Entretien avec Desrosières. Revue Sociologies Practiques. 2011; 22: 15-18.

71. Christian, n. 70.

72. Morel, n. 7, p. 44 
obtenido después de una larga discusión de cuatro años, que exigió una secuencia de negociaciones, la elaboración de propuestas y contrapropuestas, para concluir, finalmente, en un proceso de votación. Como lo había manifestado la Société de Médecine Mentale de Belgique desde el inicio, su propuesta no era crear una clasificación científica de acuerdo a los procedimientos aceptados por la anatomía patológica o la fisiología, sino establecer, por acuerdo, una nosología aceptada por todos. Se trataba de garantizar que la inversión social destinada a la definición de esta clasificación fuera lo suficientemente robusta y confiable como para consolidar su utilización en futuros estudios estadísticos.

La clasificación se había transformado en un medio, no secundario sino indispensable, para que pudiera realizarse el deseado proceso de cuantificación y normalización, tan necesario para el proceso de internacionalización de la psiquiatría. Una buena estadística exigía mucho más que transformar hechos o palabras en números. Era necesario que las palabras se convirtieran en categorías, en clases, y ese era el desafío colectivo que habían asumido los participantes del Congrés International de Médecine Mentale de 1889.

Entonces, poco a poco, ese proceso de cuantificación que suponía la previa definición, por consenso, de enfermedades psiquiátricas como clases de equivalencia, tendió a naturalizarse y a cristalizarse. A partir de ese momento, los procedimientos de cuantificación se transformaron en rutinarios, y los convenios coyunturalmente establecidos tendieron a transformarse en una realidad indiscutible.

«Las convenciones iniciales se olvidan, el objeto cuantificado se transforma en natural, y el empleo del verbo contar vuelve mecánicamente al espíritu. Esto se mantiene como una verdad inobjetable hasta que, por razones específicas en diferentes casos, esas cajas negras son reabiertas cuando surge una controversia» ${ }^{73}$.

En el caso aquí analizado, puede observarse la cristalización de un proceso de decisión que llevó a escoger una clasificación de enfermedades mentales sobre otras, dejando en la sombra las disputas y los acuerdos necesarios que precedieron a esa definición. El informe presentado en el congreso de 1889 por J. Morel y su posterior discusión, permiten observar el privilegio concedido a los países más influyentes como Gran Bretaña

73. Desrosières, n. 65, p. 64. 
y Estados Unidos, la omisión de la delegación francesa, el triunfo de la clasificación presentada por Bélgica cuatro años antes cuando se iniciaba el debate, la descalificación de las propuestas presentadas por países menos influyentes, particularmente los de América del sur, con argumentos rápidos y despectivos.

El objetivo de este estudio ha sido analizar el momento en que se inició ese proceso de cuantificación y de internacionalización en el campo de la psiquiatría. Pues, una vez que ese proceso se transforma en socialmente válido y aceptado por todos, cuando se convierte en rutina, desaparecen las dudas y tienden a olvidarse los procesos iniciales ${ }^{74}$ de negociación.

Hemos intentado poner en evidencia las disputas y mediaciones que antecedieron a la definición de una lista, en apariencia simple, de enfermedades mentales. El congreso analizado, puede ser leído como un marco de referencia para los procesos de cuantificación de la psiquiatría. En 1889, la medicina mental se encontraba en un momento de crisis. La confianza depositada en la psiquiatría clásica de Pinel y Esquirol, hacía tiempo que había desaparecido, y la teoría de la degeneración no se había impuesto como teoría hegemónica ${ }^{75}$ ni en la Société Médico-psychologique de París.

Las diferentes instancias de validación científica en las que se esperaba encontrar legitimidad fracasaban y no parecía haber criterios confiables para establecer las clasificaciones. En ese contexto, las estadísticas podían ser vistas como una forma de legitimación de las nosologías. Como afirma Desrosières:

«Los objetos cuantificados nacen en momentos de crisis, entre interacciones y luchas sociales, luego ellos viven sus vidas, circulan, se robustecen, cambian de sentido, ganan independencia. El gobierno por los números (la gestión de la locura) se apoya en estos objetos, y es eso lo que justifica que los estudiemos, no en tanto reflejos de la realidad, sino como agentes de transformación de la realidad» ${ }^{76}$.

74. A partir del momento en que el procedimiento aquí analizado permitió legitimar diversos estudios de estadística psiquiátrica posteriores, resulta cada vez más difícil que sea sometido a debate y discusión, transformándose, poco a poco, en incontestable. Christian, n. 70

75. Campos, Ricardo; Martínez, José; Huertas, Rafael. Los ilegales de la naturaleza. Medicina y degeneracionismo en la España de la Restauración (1876-1923). Madrid: CSIC; 2000.

76. Desrosières, n. 65, p. 16. 
Como vimos, para que pueda iniciarse un proceso de internacionalización de la medicina mental fue necesario estandarizar una nomenclatura que, en ese momento, pudiera servir de base para la construcción de una estadística unificada de enfermedades mentales. Así comenzó a cristalizarse y naturalizarse la introducción de la cuantificación en el campo de la psiquiatría y el uso de la estadística como instancia de legitimación de la medicina mental. Más tarde, ese procedimiento, del mismo modo que los objetos evocados por Desrosières, vivirá su propia vida, circulará, se robustecerá, cambiará de sentido y ganará independencia.

\section{Agradecimientos}

Agradezco al profesor Gerard Jorland la atenta lectura de la primera versión de este escrito. 
Classifications, agreements and negotiations: bases for the first international statistics on mental diseases (Paris 1889)

1.- Introduction. 2.-Proposals for a unified classification. 3.-Bases for international statistics on the mentally ill. 4.-Quételet and international 
statistics. 5.-Counting and quantifying. 6.-Crystallisation of a new method for classifying mental diseases.

ABSTRACT: This article discusses the construction of the first international classification of mental diseases, specifically created to allow a unified statistics on the mentally ill. This classification was discussed and approved at the International Congress of Mental Medicine held in Paris in August 1889, in response to a request made by the Society of Mental Medicine of Belgium four years earlier. A careful reading of this report helps us to understand the role of the classification and statistics of pathologies in the field of psychiatric medicine. This offers an historical perspective on an issue that remains problematic and allows analysis of the time when psychiatric classifications were first articulated and subjected to comparative statistical study. 